\title{
Leveraging Mobile Health Technology and Multidisciplinary Methodology to Optimize Self-Management Education for Advanced Cancer Pain: Development of STAMP
}

\author{
Desiree Azizoddin, PsyD \\ Dana Farber Cancer Institute \\ Brigham and Women's Hospital \\ Harvard Medical School \\ DRAzizoddin@bwh.harvard.edu
}

\author{
Rosalind Adam, MBChB, MRCGP, \\ $\mathrm{PhD}$ \\ Institute of Applied Health Sciences \\ University of Aberdeen \\ Rosalindadam@abdn.ac.uk \\ Andrea Enzinger, MD \\ Division of Population Sciences \\ Dana-Farber Cancer Institute \\ Harvard Medical School \\ Andrea_Enzinger@dfci.harvard.edu
}

\author{
Dani Kessler, BA \\ Division of Population Sciences \\ Dana-Farber Cancer Institute \\ Danifkessler@gmail.com
}

\begin{abstract}
Pain continues to be a primary and challenging symptom of cancer. We sought to use mobile health (mHealth) technology to tailor psycho-education to better meet patients' needs. Using the Agile and mHealth Development and Evaluation Frameworks, a multidisciplinary team of clinician researchers, patients, and software and design specialists followed a four-phase iterative process to develop multi-media cancer pain education within a patient-facing smartphone application. The resulting application pairs comprehensive cancer pain education spanning pharmacologic and behavioral support with medication hosting and symptom surveys. MHealth enables creative, interactive educational approaches utilizing written text, graphics, animated videos, quizzes, audiorecordings, and motivational messages. Computable algorithms were used to tailor content to patients' symptom surveys. Cancer patients found the materials to be useful. By bridging technology and research methodology, we incorporated theory, evidence, and patient feedback to create a tailored and scalable educational intervention to support cancer pain selfmanagement.
\end{abstract}

\section{Introduction}

Pain affects $75-90 \%$ of patients with advanced incurable cancer [1], and has a major impact on quality of life. Opioids are considered the standard treatment for moderate to severe cancer pain [2]; however, many patients experience poorly controlled pain despite having access to opioids [3], [4].

Patients frequently lack the knowledge, selfmanagement skills, and support needed to utilize opioids and manage their pain effectively [5]. To meet these needs, psychoeducational interventions have been extensively evaluated for cancer pain [6], [7]. Unfortunately, meta-analyses suggest that psychoeducational cancer pain interventions have yielded only small benefits to pain severity and functional wellbeing [6]-[8]. This limited efficacy may relate to shortcomings in existing interventions, the majority of which have delivered static cancer pain education via booklets or videos, sometimes augmented by one or a few structured clinician interactions [7]. Although some interventions provide opportunities for active learning, few have tailored education or offer longitudinal support that address the dynamic nature of the cancer pain experience[5], [9]-[12]. Moreover, few educational interventions address underlying psychological and behavioral processes that influence pain self-management, such as motivation, stress management, self-efficacy, and addiction fears within the cultural context of the opioid epidemic [3], [4], [9], [10].

Mobile health (mHealth) technology is a promising yet underutilized strategy to tailor and deliver cancer pain psycho-education to patients in their home environment and to harmonize education with support for other critical aspects of self-management such as medication organization, supporting care team communication, and offering real-time symptom management advice. Most mHealth apps developed for cancer pain have simply facilitated pain reporting 
without robust or tailored psychoeducation [12]-[14] or have focused on patients with early stage cancers or in survivorship.[15] A few others have taught behavioral pain management strategies via pre-recorded lessons or video chat with a therapist, but have had limited support for the medical aspects of self-management [11], [16], [17]. Existing mHealth symptom support interventions have not developed to address the inherent differences present in the management of advanced cancer pain. Additionally, existing mHealth strategies have not fully capitalized upon the technology to personalize symptom education and support (i.e. using computable algorithms, or patient-facing clinical decision support) to treat cancer pain [11], [17]. There has also been little emphasis on exploiting creative, user-centered design possibilities to enhance the impact of the education itself [12], [18].

A critical barrier to providing symptom education through technology is the scant literature describing experiences and methodologies for developing quality mHealth psycho-educational applications, and few integrate patients' perspectives - the target population [11], [19]-[21]. The Smartphone Technology to Alleviate Malignant Pain (STAMP) study aims to optimize opioid management for advanced cancer pain through mHealth technology. Here we describe a rigorous, reproducible methodology to develop comprehensive cancer pain psycho-education for delivery via an mHealth application. Our objective was to develop comprehensive cancer pain education materials formatted for mHealth, and to leverage the unique potential of technological solutions to tailor and deliver that education to patients in a way that is responsive to their pain management needs in the moment.

\section{Methods}

\subsection{Procedures for development}

We combined the Agile [22] and mHealth Development and Evaluation Framework [19] models to develop our application. Both are well established, highly iterative software development frameworks built upon real-time collaborations with interdisciplinary teams and participant feedback in each phase of intervention development [19], [22], [23]. These methodologies emphasize being guided by the existing literature and building upon theoretical models that align with anticipated mechanisms of action.[24]-[26] These models also emphasize the importance of interdisciplinary collaborations that include content experts, technologic and design experts, and target users. Modeled after the mHealth Development and
Evaluation Framework,[23] our content and application development was carried out in four, pragmatic phases including: 1) Defining the theoretical and conceptual basis, 2) Refining concepts through formative research, 3) Developing and optimizing content, and 4) Refining intervention content. Borrowing from the Agile framework,[19] STAMP development involved the target population and key stakeholders (researchers, software and design partnerships, patients, and clinicians) to employ rapid cycles of content review and feedback that drive iterative, responsive-to-change development procedures within and between each phase. See Table 1 and Figure 1 for complete development methodology. This study was approved by the Dana-Farber Cancer Institute (DFCI) Institutional Review Board.

\subsection{PHASE 1: Defining the theoretical and conceptual basis of STAMP}

Grounded in Wagner's Chronic Care Model (CCM) [27], the underlying goal of STAMP is to use mHealth to support both advanced cancer patients and care teams in the management of cancer pain in the outpatient setting. CCM emphasizes redesign of reactive, acuteepisode-oriented approaches to care delivery, in favor of proactive interactions between activated/informed patients, and prepared/proactive care teams.

2.2.1. Establishing a primary study team and conceptualizing the intervention. The Primary Study Team included content experts in oncology, palliative care, pain psychology, and nursing - many of whom also had expertise in health information technology and cancer care delivery research. At the outset of the project, the primary team met several times to discuss and refine the overarching goals of the project, to map these goals onto a set of application features, and to discuss the overall vision and "feel" of the application. We then had a series of meetings with a digital media consultant, and with a team of software programmers to scope the application and prioritize features, modifying and eliminating complex or costly aspects.

\subsection{PHASE 2: Refining concepts through formative research}

2.3.1. Engagement of patient and professional stakeholders. To further guide development of the application, we recruited members for a Patient Advisory Panel by partnering with the Patient Family Advisory Council of a large academic cancer center, and by soliciting referrals from clinicians at that center. Patients were invited if they had personal experience 
with cancer pain and had used opioids. We also formed a Clinician Advisory Panel by recruiting a diverse group of clinicians and researchers with expertise in cancer pain management. Specifically, we sought participation of physicians, nurses, pharmacists, and psychologists practicing within the fields of medical oncology, palliative care, and primary care, seeking out those who had previous experience developing tech interventions.

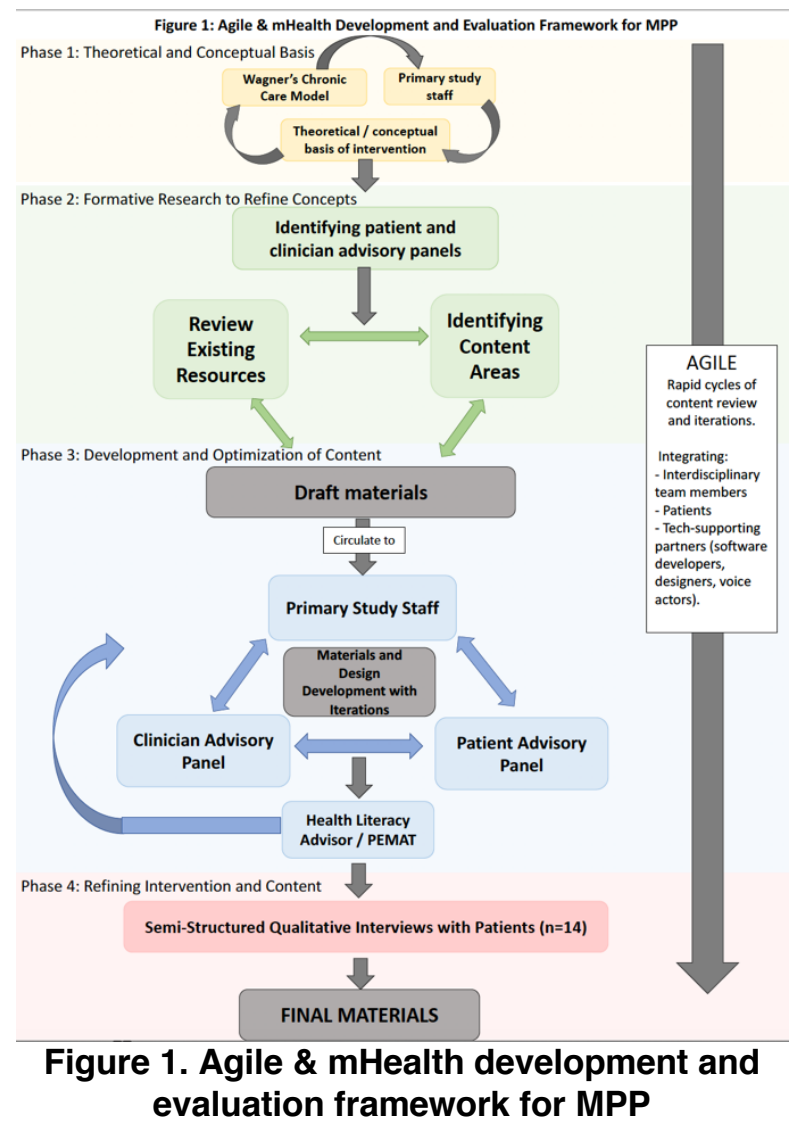

2.3.2. Stakeholder engagement. Clinician and patient advisors were oriented to the project goals from the initial stages. Thereafter, the clinician advisory panel met in biweekly working groups dedicated to defining priority content, brainstorming, reviewing and revising draft materials, and providing feedback on formatting, visual design, and media production. The patient advisory panel met with the primary research team quarterly to review content and provide feedback to meet the needs of the target population.

2.3.3. Review of existing cancer pain education. To identify priority content areas, we reviewed patient education materials available online through leading international oncology organizations[28]-[32]. We also reviewed publications of cancer pain psychoeducational interventions for their descriptions of educational content [7]. The team indexed this data and created a spreadsheet of potential topics for inclusion, including examples from existing materials.

2.3.4. Selection of priority content areas. The clinician advisory panel reviewed the list of candidate educational topics through iterative discussions, identifying topics to eliminate or add, and suggesting areas that were important to deepen or refine based upon their clinical expertise. The clinician advisory panel focused on commonly-noted self-management challenges and questions frequently asked by patients. Patient advisors also reviewed the content priority areas, sharing personal reflections and highlighting information they "wished they knew earlier on."

\subsection{PHASE 3: Developing and optimizing content}

\subsubsection{Selecting formats for mHealth educational} content to enhance user engagement. The primary study team, graphic designers, and clinician and patient advisory panels discussed formatting options for educational content, including two dimensional (2-D) animated videos, long-form text, shorter texts (e.g. teaching pearls), medication-specific education, quizzes, animated visuals, and audio-recordings. A matrix was created to decide which content areas would be presented in which format(s), with the most important content presented in multiple formats.

2.4.2. Development of educational content. Core members of the primary study team and a digital media consultant met regularly to draft and refine novel written content. The team prioritized a conversational and empathic tone [33], as opposed to clinical or formal styles of writing. The primary team and advisory panels also brainstormed creative ways to present conceptually difficult information, including analogies, metaphors, and supporting visuals. The pain psychologist evaluated evidence-based relaxation exercises and developed scripts with modified content for cancer pain. For comprehensive medication teaching, the primary team identified core information for patients' medication needs, vetted validated systems and reformatted content using patient-centered language. Concepts and scripts for the videos and relaxation exercises were developed through a group writing process with integral involvement from the digital media consultant and the clinician and patient advisory panels.

2.4.3. Production of educational content. Written materials were developed using best practices [34], including targeting a $6^{\text {th }}-8^{\text {th }}$ grade reading level, using 
headers and bulleted lists, defining medical terms, and creating content summaries and action steps. We used a web-based content-management system to create a userfriendly smartphone display with color-coded headers, accordion graphical control elements (i.e. collapsible/expandable lists), paired visuals, and hyperlinks to cross-reference related materials as seen in previous reviews to be related to patients perspectives for health related information.[35]

Prioritizing strong visuals and creative information display, we collaborated with a digital media consultant and a team of artists to develop animated characters to be featured within a set of 2-D animated videos and across the application. Characters were designed to be warm and relatable, without specific gender or racial hallmarks, yet still registering as human (i.e. having two legs/arms). After our media specialist mocked-up storyboards, the scripts were recorded by a professional voice actor and ultimately animated into $2 \mathrm{D}$ films. The team brainstormed strategic areas the character could be integrated across the application. The ADK-software designers integrated the content with paired characters/visuals into wireframes that were reviewed by the advisory panels. See Figure 2 .

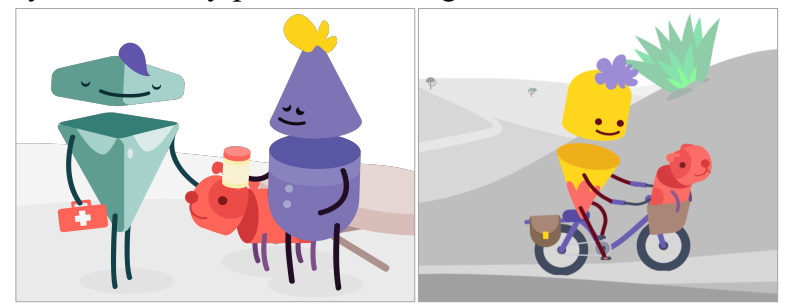

Figure 2. Examples of final application characters

2.4.4. Stakeholder review, quality assurance, and iterative content refinement. Each production process involved iterative rounds of feedback from the study team and advisory panels between each step. The clinician advisory panel reviewed and iteratively revised all content during the biweekly meetings to ensure that they were clear, accurate, useful, and actionable for patients [34]. Once drafts were nearly finalized, the clinician advisory panel systematically rated them using the 24-item Patient Education Materials Assessment Tool-Printable materials (PEMAT-P) [36], [37]. Scores are totaled to provide understandability scores $(\%)$ and actionability scores (\%), where higher scores were better. We used the "Health Literacy Advisor" software program to assess content and assign a Fry-based grade level score for literacy level [38], with the goal of meeting a $6-8^{\text {th }}$ grade reading level [39]. The patient advisory panel also reviewed select content during quarterly meetings and provided feedback, although they were not asked to score them using PEMAT.

\subsection{PHASE 4: Refining intervention content}

2.5.1. Patient feedback and revisions. Following phases 1-3, we obtained feedback from our target population. The DFCI IRB reviewed this activity and considered it to be exempt. Eligible patients were adults with an advanced cancer who had used short- and longacting opioids for cancer-related pain. Exclusion criteria included cognitive impairment, inability to speak English, and opioid use disorder. A trained interviewer (D.A. and D.K.) conducted in-person, semi-structured individual interviews using standard cognitive interviewing techniques (think alouds, rephrasing) to assess the clarity of the content, supplemented by additional questions to assess usefulness and suggested improvements. Participants reviewed and provided feedback on wireframes, design concepts (e.g. characters), and audiovisual content that were presented through a webpage on an iPad, via secure video-chat, or in print before during their interview. Interviews were audio-recorded to assist with note-taking and lasted 3060 minutes. Notes were then reviewed using content and thematic qualiatiative analysis methods[40] to inform content revisions. Exemplary questions for patient interviews included: "Can you explain in your own words what this is asking?"; "Was anything confusing, if so, what was?"; "were there any words or phrases that were unfamiliar to you?" Patients were also asked about their experiences of using a $0-10$ scale to report their pain and perceptions and utlity of the ratings the acceptability of their pain. Patients were also asked about their experiences of using a 0-10 scale to report their pain and perceptions and utlity of the ratings the acceptability of their pain.

\section{Results}

\subsection{Patient and professional stakeholders}

The multidisciplinary clinician advisory panel included 11 specialists with experience in health-related technology from palliative care, medical oncology, pain psychology, nursing, palliative care pharmacy, primary care, and patient education. Six patient advisors joined the panel and attended quarterly meetings.

\subsection{Key components of the STAMP application}

After a process of refining priorities for the application (section 2.2), we agreed upon the following set of core application features: 1) a resource library 
with comprehensive multi-media symptom education, 2) a virtual "medicine cabinet" to organize and provide specific teaching for patients' analgesics and laxatives (which are important for managing opioid-induced constipation), 3) daily symptom and medication reporting, 4) delivery of tailored educational/ motivational messages driven by patients' reported symptoms, 5) clinical decision support elements to provide patients with specific advice for managing laxatives, and 6) a web-based clinician portal that presents patients' symptom data and facilitates proactive outreach and communication.

\subsection{Review of publicly available cancer pain education and determination of priority content}

Review of publicly available cancer pain educational materials found that most included introductory explanations of cancer pain (neuropathic, bony), treatment options (opioids, nonopioids, interventional pain procedures, complementary approaches), opioid formulations (oral, parenteral, transdermal), opioid side effects, and suggestions for communicating symptoms to care teams. Available resources were text-heavy, written from a clinician perspective without specificity to patients' viewpoints with few supportive visuals [7]. Most resources provided broad overviews of the aforementioned topics, but lacked specificity and actionable advice patients need to self-manage (e.g. how to take your breakthrough opioid, when to expect relief or resurgence of pain, suggestions for managing over-the-counter laxatives). Finally, few resources explained psychological or behavioral contributors to pain, and non-pharmacologic pain management strategies were limited to lists of options (e.g. yoga, reiki) without any information on how to access or learn these strategies [7].

There was a strong consensus from patient and clinician advisory panels that integrating opioid and psychological education about pain [41], [42] in one resource with patient-centered content and advice could be particularly helpful to patients. In an effort to tailor medication content to patients' specifics needs, we included only pain and laxative medications that would be pertinent to each patient, as opposed to comprehensive lists of medications. We also integrated biopsychosocial aspects of pain management throughout all content and focused on providing patientcentered recommendations with action items throughout app content and live exercises (e.g. relaxation recordings).

\subsection{Final educational content}

In total we developed 28 long-form texts with supportive visuals, 11 quizzes to highlight common selfmanagement challenges (paired with visuals), a suite of 108 brief educational and/or motivational messages, 3 animated videos (add all video links here), comprehensive information for 34 distinct medications, and 12 audio-recorded relaxation exercises. Table 1 presents an overview of final educational content areas and formats.

Specifically, content topics combined an emphasis on pharmacologic and non-pharmacologic aspects of pain self-management including medication support (e.g. using short and long-acting opioids, managing side effects, opioid safety); pain psychology (e.g. pain perception, pain and the stress response); health behaviors and pain (e.g. sleep hygiene); skills training (e.g. activity pacing, relaxation recordings); and constipation advice (e.g. laxative regimens). See Figure 3 for example long-form text.

\section{Table 1. Review of education topics and multi-} media delivery formats throughout MPP

\begin{tabular}{|c|c|c|c|c|c|c|}
\hline Educational Materials & \begin{tabular}{|c|} 
Long- \\
form \\
texts w/ \\
visuals \\
\end{tabular} & $\begin{array}{c}\text { Bite- } \\
\text { sized } \\
\text { tips }\end{array}$ & Videos & $\begin{array}{c}\text { Scenarios } \\
\text { / Quizzes }\end{array}$ & $\begin{array}{c}\text { Med } \\
\text { Specific }\end{array}$ & $\begin{array}{c}\text { Relaxation } \\
\text { scripts }\end{array}$ \\
\hline \multicolumn{7}{|l|}{ Opioids } \\
\hline Understanding Opioids & $\mathrm{x}$ & $\mathrm{x}$ & $\mathrm{x}$ & $\mathrm{x}$ & & \\
\hline Opioids for Breakthrough Pain & $\mathrm{x}$ & $\mathrm{x}$ & $\mathrm{x}$ & $\mathrm{x}$ & $x$ & \\
\hline Long-Acting Opioids & $\mathrm{x}$ & $\mathrm{x}$ & & $\mathrm{x}$ & $\mathrm{x}$ & \\
\hline \begin{tabular}{|l} 
Opioids: Which One Do I Take? \\
\end{tabular} & $\mathrm{x}$ & $\mathrm{x}$ & $\mathrm{x}$ & $\mathrm{x}$ & & \\
\hline Using Opioids Safely & $\mathrm{x}$ & $\mathrm{x}$ & & $\mathrm{x}$ & & \\
\hline Addiction Worries & $\mathrm{x}$ & & & & & \\
\hline Managing Opioid Side Effects & $\mathrm{x}$ & $\mathrm{x}$ & & & & \\
\hline What is Naloxone? & $\mathrm{x}$ & & & & & \\
\hline $\begin{array}{l}\text { Medication Education: opioid pain } \\
\text { medications }\end{array}$ & & & & & $\mathrm{x}$ & \\
\hline \multicolumn{7}{|l|}{ Non-opioid pain medications } \\
\hline $\begin{array}{l}\text { Medication Education: non-opioid } \\
\text { pain medications }\end{array}$ & & & & & $\mathrm{x}$ & \\
\hline \multicolumn{7}{|l|}{ Opioid-Induced Constipation } \\
\hline \begin{tabular}{|l|} 
Medication Education: Laxatives \\
\end{tabular} & $\mathrm{x}$ & $\mathrm{x}$ & & $\mathrm{x}$ & $\mathrm{x}$ & \\
\hline Managing Constipation & $\mathrm{x}$ & & & $\mathrm{x}$ & & \\
\hline Understanding Laxatives & $\mathrm{x}$ & & & $\mathrm{x}$ & & \\
\hline Staying Regular with Laxatives & $\mathrm{x}$ & & & & & \\
\hline $\begin{array}{l}\text { When Constipation Gets Bad: Your } \\
\text { Rescue Plan }\end{array}$ & $\mathrm{x}$ & & & & & \\
\hline \multicolumn{7}{|c|}{ Big Picture of Pain (Pain and Mind-Body) } \\
\hline Understanding Pain & $\mathrm{x}$ & $\mathrm{x}$ & & & & $x$ \\
\hline Emotions and Pain & $\mathrm{x}$ & $\mathrm{x}$ & & & & $\mathrm{x}$ \\
\hline Stress and Pain & $\mathrm{x}$ & $\mathrm{x}$ & & & & $\mathrm{x}$ \\
\hline The Bigger Picture of Pain & $\mathrm{x}$ & $\mathrm{x}$ & & & & \\
\hline \multicolumn{7}{|l|}{ Living with Pain and Health Behaviors } \\
\hline Why Goals Matter & $\mathrm{x}$ & $\mathrm{x}$ & & & & \\
\hline Why Report Your Pain? & $\mathrm{x}$ & & & & & \\
\hline $\begin{array}{l}\text { Staying active with pain: activity } \\
\text { pacing }\end{array}$ & $\mathrm{x}$ & $\mathrm{x}$ & $\mathrm{x}$ & & & \\
\hline Better Sleep, Better Pain & $\mathrm{x}$ & $\mathrm{x}$ & & & & \\
\hline \begin{tabular}{|l} 
Pain Coping Tools \\
\end{tabular} & $\mathrm{x}$ & $\mathrm{x}$ & & & & \\
\hline \multicolumn{7}{|l|}{ Communication } \\
\hline Communicating About Pain & $\mathrm{x}$ & $x$ & & & & \\
\hline TOTAL & 22 & 108 & 3 & 11 & 34 & 11 \\
\hline
\end{tabular}

Each of the three videos (two-minutes long) supported core concepts of self-management [43], [44]. The first two reviewed primary principles of opioids and pain management (addressing ambivalence and dosing variations) and the third reviewed activity pacing to achieve meaningful goals. We integrated metaphors to promote understanding throughout educational cotent, for example to clarify the differences between short and long acting opioids in video animations, we used an 
analogy centered around riding a bicycle on a hilly landscape. We used another analogy of acclimating to the temperature of a warm bath to explain opioid tolerance and withdrawal and target patients' concerns about opioid addiction. All content was scored above an $80 \%$ level of actionability and understandability using the PEMAT, except one section in a text about opioid dosing. (Table 2). This was due to the fact that the opioid content did not include a summary of instructions for dosing or titating opioids, as this was not advisable for patients' safety. All text met a $7^{\text {th }}$ grade, Fry-based reading level [38].

\section{Table 2. PEMAT scoring for all long-form} educational content

\begin{tabular}{lcc}
\hline & $\begin{array}{c}\text { Understandability } \\
\text { score (\%) }\end{array}$ & $\begin{array}{c}\text { Actionability } \\
\text { score (\%) }\end{array}$ \\
\hline Opioid Basics & 95.5 & 57.89 \\
Opioid Side Effects & 87.9 & 100.0 \\
Opioid Safety & 91.6 & 97.2 \\
Constipation \& & 100 & 100.0 \\
Laxatives & 93.7 & 88.2 \\
Understanding pain & 98.1 & 95.2 \\
Emotions \& Pain & 89.0 & 85.7 \\
Sleep & & \\
\hline
\end{tabular}

\subsection{Linking self-monitoring with personalized educational content and feedback}

In an effort to get relevant information to patients in their moments of need, we developed several strategies for information delivery through Key Components 1, 2, and 4 listed in Section 3.2 including the: Resource library - all long-form content, videos, and relaxation recordings were available, "Medicine Cabinet" - medication-specific teaching where patients only received education related to prescription or overthe counter medicines they were actually taking, and Tailored education algorithms with "bite-sized tips" of brief education delivered to patients' in response to their daily symptom reports.

Following completion of daily symptom reports (Section 3.2- Key component 3), patients received a survey summary that included tailored education and symptom-management advice for pain, constipation, and opioid side effects. Computable algorithms driven by 3 pain-related survey items (best pain, worst pain, and pain acceptability) classified patients as having "good," "suboptimal," and "poor" pain control that day. (See Table 3 for algorithm logic). In line with previous literature[45], [46], pain processing and reporting is highly variable and multi-dimensional. To accurately delineate patients' pain management needs in the moment, we sought to identify methods that would allow the technology to better decipher patients' needs and provide more accurate advice. Following patient cognitive interviewing, an item exploring the level of acceptability for patients pain in the moment was determined as an appropriate solution.

\section{How your brain and nerves work together to feel pain \\ Pain happens when nerves are triggered. Let's say you bump your elbow. The signal travels from your elbow, through your nerves to your brain, and then you feel pain. A similar thing can happen when you have a tumor.

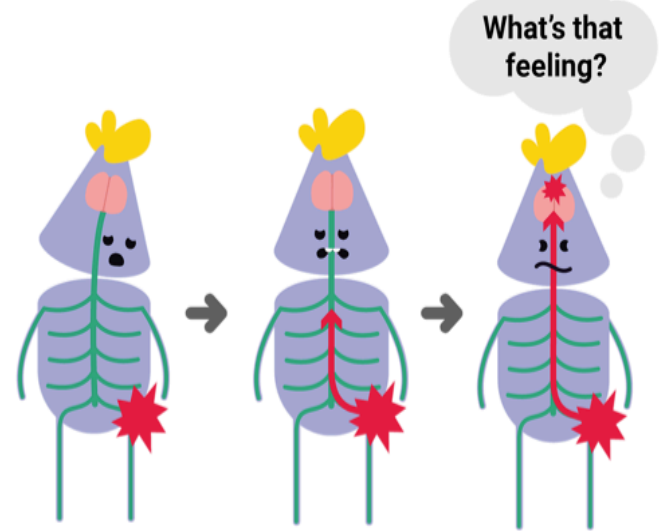 \\ Signals about pain travel to both the physical and the emotional parts of your brain. That's why being in pain not only hurts physically, but also emotionally.
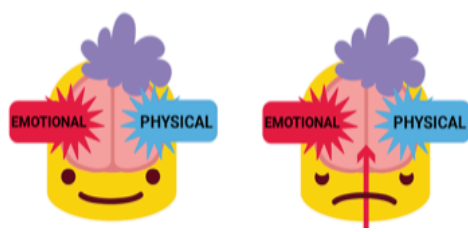 \\ Figure 3. Example long-form text with visuals that review cancer pain perception}

Patients would receive a brief psycho-educational message tailored to this classification, which was randomly selected from a library of (40-50 messages per category) spanning topics of insight-building, motivating toward pain-management goals, pharmacologic education, pain psychology principles (e.g. understanding stress and pain), and learning relaxation strategies. Each message began with an empathic statement suited to their level of pain control, and was paired with a character image mirroring good, suboptimal, or poor pain control. Each brief message linked to more comprehensive education housed within the resource library. Tailored feedback for constipation used clinical decision support algorithms to generate specific advice regarding titrating over-the-counter laxatives, and when to contact care teams. Together, these methods of content engagement, the tailored education and content library, would strive to increase patients' accessibility to promote self-management and reinforce patients' learning in moments of need.

\subsection{Integrating patient feedback into the intervention}


Patient feedback was integrated through two processes: 1) the patient advisory panel throughout application and content development, and 2) individual interviews with patients from our target population enrolled to the study to review wireframes and content (Section 2.5.1).

\section{Table 3. Pain score (average and worst pain) and acceptance algorithms and categories for personalized educational content and feedback}

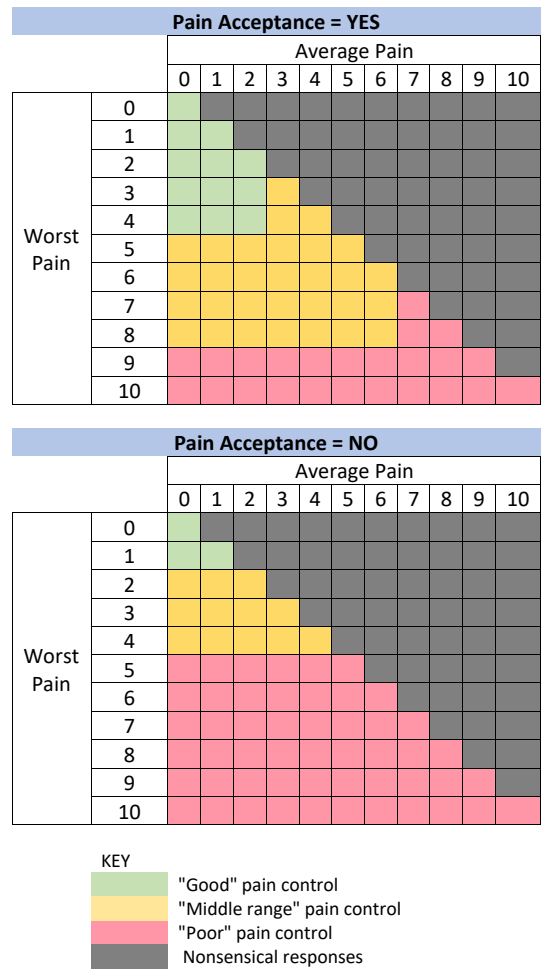

Note: Pain items included: "In the past 24 hours, what was your pain on average? \& In the past 24 hours, what was your pain at its worst?" - Numeric Rating Scale 0-10) and the acceptability report: "Pain is never a good thing, but in the past 24 hours has your pain been acceptable to you?" - "yes" or "no."

The patient advisory panel $(\mathrm{n}=6)$ appreciated the comprehensive and patient-centered nature of the educational content. They requested "more realistic" examples of physical activities within behavioral goalsetting topics (i.e. exercising/bicycle riding was considered too difficult, and instead recommended knitting, reading, or walking). Notably, patient stakeholders voiced significant concerns around addiction as a deterrent to opioid use. In response, we included comprehensive education explaining opioid tolerance, dependence and addiction, integrating metaphors with visuals to exemplify tolerance. Overall, the patient advisory panel underscored their favorable views of the simplicity, tone, and patient-friendly language of the content, the visuals and metaphors made principles easier to grasp, and that the biopsychosocial framework validated their opioid use.

After content revisions, we enrolled 14 patient who were not previously oriented to the app and reviewed wireframes of the content and its delivery. A few patients requested that sections of the opioid education be shortened, leading to the creation of subsections of separate opioid education. Patients were particularly receptive to the visuals and the amount of content presented on wireframes. One patient explained, "I like the amount of verbiage you have on here. ...It's informative enough that I'm going to look at it and go, 'Oh I'm going to read this.' I like having the separate pages and smaller pieces to read, than I would a larger document. So, user-friendliness is great." Patients described learning new content after reviewing the longform text and videos and asked to take the "useful" materials home. A few patients requested that the font be larger and darker on wireframes of the content and modifications were made accordingly. They described the algorithmic-advice including bite-sized tips, longform educational content, and videos as being "helpful" and "a great reminder."

In summary, one patient stated, "It makes sense and I think people need it...It's wicked hard, and I think they can benefit from this." Overall, patients were receptive to the content and methods for interaction, identifying the content to be "well-written," "relatable," and "accurate," and all together acceptable, user-friendly, and informative.

\section{Discussion and Conclusion}

\subsection{Discussion}

The authors share reproduceable, research methodology that applied frameworks borrowed from technology development strategies to create a suite of user-centered cancer pain education within an mHealth application for advanced cancer patients with pain. Partnering with software developers, media designers, specialty clinicians, researchers, and patients, we employed a joint methodological approach that incorporated theory, evidence, and knowledge from key stakeholders using the Agile[22] and the mHealth Development and Evaluation Frameworks[19]. By bridging technology and research methodology relevant to technology and health behavior change theories, we were able to develop a rapidly evolving intervention geared to suit the needs of our primary audience patients dealing with advanced cancer pain. We created extensive cancer pain educational content presented through several multimedia formats and integrated with 
patients' daily symptom reports to capitalize on app design and promote patient engagement[12], [18], [35].

Many cancer pain educational interventions lack an interdisciplinary approach, patient-centered advice, and engaging media integration.[7] Currently available mHealth apps to support cancer pain include static educational content presented through modules and simple pain reporting,[11]-[14], [16], [17] without integrating tailored education. To improve accessibility, we used the Agile[22] and the mHealth Development and Evaluation Framework[19] that engender interactive materials as opposed to static, booklet-based educational materials that are often overlooked by patients[7]. We sought to fill these gaps and create a more engaging collaborative mHealth application for patients by tailoring education through three primary avenues, 1) incorporating multimedia and visually engaging content, [11], [17] 2) various formats for education materials, and 3) pairing education in response to patient-reported pain algorithms. Through multidisciplinary efforts and innovative technology taking from health behavior change principles, we developed extensive patient-centered education presented in multimedia formats including 2D animated videos, interactive long form text with supportive visuals, quizzes, brief messages, and audio recordings. The unique methodology used to develop this application fostered innovation in how education would be delivered to patients. Specifically, STAMP uses pain reporting algorithms to deliver diverse advice and paired education to lend patients tangible tools in response to their current symptoms and reduce being overwhelmed with large amounts of information.

Patients consistently identified tailored algorithms that drive education sharing in their moment of need as a strength of the intervention, although feasibility, acceptability, and effectiveness need to be evaluated in future trials.

Another key objective was to match advanced cancer patients' needs with best practices and evidencebased pain management using technological solutions. To our knowledge, this is the first mHealth app that combines education relevant to opioids, patients' unique barriers to opioids, constipation and side effect management, pain processing, psychological factors related to pain, and activity-based goals. In addition to creating comprehensive, evidence-based education, all content utilized best health communication practices [34] with text at a $7^{\text {th }}-8^{\text {th }}$ grade reading level, reduced clinical jargon, and emphasized the patient-perspective by including actionable self-management items throughout. Patients especially appreciated the medication specific education that was more understandable, the integration of the biopsychosocial approach to pain, and the clear guidance to improve self- management strategies throughout their day-to-day life. Overall, patients approved the content and appreciated the tone, overall simplicity, and integrative education available through the application.

Although mHealth is an increasingly popular approach for patient interventions, processes and frameworks to guide their development are lacking[19], [20]. Technology companies, health service providers, behavioral scientists, commercial organizations, and designers all have their own approaches to mHealth development and often have different agendas. Despite technology's great potential, many mHealth projects fail because of challenges anticipating and balancing theoretical, clinical, and technical needs. These projects require ongoing trans-disciplinary cooperation and structures to facilitate timely exchanges to achieve realtime alignment of goals and adaptation[22]. Integrating a multidisciplinary team of researchers, clinicians, and technology experts with patients input from initial stages and throughout development allowed us to overcome these common barriers to successfully create a unique mHealth cancer pain application.

The STAMP application will be tested in a single arm pilot study to to confirm the feasibility, acceptability, and utility of this novel mHealth selfmanagement system for a highly symptomatic population, patient with advanced cancer and complex pain self-management needs. We will explore intervention effects on patients' pain outcomes (pain intensity/interference) and secondarily opioid use, psychological wellbeing, physical function, and care engagement.

The demographic makeup of patients treated at this urban cancer center may serve as a potential limitation to the generalizability of their feedback. It may also be the case that patients provided overly positive critiques to not be harsh to the researcher and developers who were involved in both development and conducting the interviews. Due to expense limitations, content algorithms relied solely on pain, though future iterations would also strive to include medication reporting and stress in advice algorithms to immediately target patients' multi-faceted pain needs. The current mHealth application is not yet available to the public.

\subsection{Conclusion}

The development and production methodology of STAMP presented in this manuscript can serve as a successful example of joint scientific research, clinical, patient, and technological collaborations to treat a complex medical symptom - advanced cancer pain. Through the collaboration of stakeholders with variable expertise, we were able to create a host of patientcentered, multi-media educational materials in an 
mHealth app to support patients with advanced cancer pain. Educational materials not only reviewed a wide range of pain, opioid, constipation, and pain psychology related needs, but also did so through interactive, algorithm-based technology.

Pain continues to be a primary and challenging symptom of cancer, especially in advanced cancer. Novel interventions for cancer pain must be patientcentered, be accessible at home, and integrate with patients' current treatments including opioids. This mHealth application provides tailored pain management education and advice, and combines relevant topics on pain, opioid, and self-management. If proven successful, this app could improve advanced cancer patients' experiences with pain, pain-related clinic appointments and hospitalizations, and make cancer pain management more widely accessible.

\section{References}

[1] M. H. J. Van Den Beuken-Van Everdingen, L. M. J. Hochstenbach, E. A. J. Joosten, V. C. G. Tjan-Heijnen, and D. J. A. Janssen, "Update on Prevalence of Pain in Patients with Cancer: Systematic Review and Meta-Analysis," J. Pain Symptom Manage., vol. 51, no. 6, pp. 1070-1090.e9, 2016.

[2] World Health Organization, WHO guidelines for the pharmacological and radiotherapeutic management of cancer pain in adults and adolescents. 2018.

[3] J. H. Kwon, "Overcoming barriers in cancer pain management," J. Clin. Oncol., vol. 32, no. 16, pp. 1727 1733, 2014.

[4] R. Jacobsen, C. Møldrup, L. Christrup, and P. Sjøgren, "Patient-related barriers to cancer pain management: A systematic exploratory review," Scand. J. Caring Sci., vol. 23, no. 1, pp. 190-208, 2009.

[5] S. Dalal, K. C. Tanco, and E. Bruera, "State of art of managing pain in patients with cancer," Cancer J., vol. 19,

[6] M. I. Bennett, A. M. Bagnall, and S. José Closs, "How effective are patient-based educational interventions in the management of cancer pain? Systematic review and metaanalysis," Pain, vol. 143, pp. 192-199, 2009.

[7] R. Adam, C. Bond, and P. Murchie, "Educational interventions for cancer pain. A systematic review of systematic reviews with nested narrative review of randomized controlled trials," Patient Educ. Couns., vol. 98, no. 3, pp. 269-282, 2015, doi: 10.1016/j.pec.2014.11.003.

[8] R. H. Dworkin et al., "Core outcome measures for chronic pain clinical trials: IMMPACT recommendations," Pain, vol. 113, no. 1-2, pp. 9-19, 2005

[9] E. M. Wright et al., "Patient patterns and perspectives on using opioid regimens for chronic cancer pain," J. Pain
Symptom Manage., 2019, doi:

10.1016/j.jpainsymman.2019.02.023.

[10] C. Zheng et al., "Benefits of mobile apps for cancer pain management: Systematic review," JMIR mHealth uHealth, vol. 8, no. 1, pp. 1-10, 2020, doi: 10.2196/17055.

[11] T. J. Somers et al., “An mHealth pain coping skills training intervention for hematopoietic stem cell transplantation patients: Development and pilot randomized controlled trial," J. Med. Internet Res., vol. 20, no. 3, pp. 116, 2018, doi: 10.2196/mhealth.8565.

[12] L. A. Jibb et al., "Development of a mHealth Real-Time Pain Self-Management App for Adolescents With Cancer: An Iterative Usability Testing Study," J. Pediatr. Oncol. Nurs., vol. 34, no. 4, pp. 283-294, 2017, doi: $10.1177 / 1043454217697022$.

[13] M. J. Allsop et al., "Multidisciplinary Software Design for the Routine Monitoring and Assessment of Pain in Palliative Care Services: The Development of PainCheck," JCO Clin. Cancer Informatics, no. 3, pp. 1-17, 2019, doi: 10.1200/cci.18.00120.

[14] S. Agboola et al., "Pain Management in Cancer Patients Using a Mobile App: Study Design of a Randomized Controlled Trial," JMIR Res. Protoc., vol. 3, no. 4, 2014.

[15] E. Hernandez Silva, S. Lawler, and D. Langbecker, "The effectiveness of mHealth for self-management in improving pain, psychological distress, fatigue, and sleep in cancer survivors: a systematic review," J. Cancer Surviv., vol. 13, no. 1, pp. 97-107, 2019, doi: 10.1007/s11764-018-0730-8.

[16] T. J. Somers et al., "A Pilot Study of a Mobile Health Pain Coping Skills Training Protocol for Patients with Persistent Cancer Pain," J. Pain Symptom Manage., vol. 50, no. 4, pp. 553-558, 2015, doi:

10.1016/j.jpainsymman.2015.04.013.

[17] T. J. Somers et al., “A Small Randomized Controlled Pilot Trial Comparing Mobile and Traditional Pain Coping Skills Training Protocols for Cancer Patients with Pain," Pain Res. Treat., vol. 2016, 2016.

[18] G. A. Wildenbos, L. W. Peute, and M. W. M. Jaspers, "A framework for evaluating mHealth tools for Older Patients on Usability," Stud. Health Technol. Inform., vol. 210, pp. 783-787, 2015.

[19] M. A. Jacobs and A. L. Graham, "Iterative development and evaluation methods of mHealth behavior change interventions," Curr. Opin. Psychol., vol. 9, pp. 33-37, 2016, doi: 10.1016/j.copsyc.2015.09.001.

[20] M. J. Allsop, S. Taylor, M. R. Mulvey, M. I. Bennett, and B. M. Bewick, "Information and communication technology for managing pain in palliative care: A review of the literature," BMJ Support. Palliat. Care, vol. 5, pp. 481

[21]C. S. Dorfman et al., "Development and pilot testing of 
an mHealth behavioral cancer pain protocol for medically underserved communities," J. Psychosoc. Oncol., vol.37, no. 3, pp. 335-349, 2019.

[22] A. Cockburn and J. Highsmith, "Agile software development: The people factor," Computer (Long. Beach. Calif)., vol. 34, no. 11, pp. 131-133, 2001.

[23] R. Whittaker, S. Merry, E. Dorey, and R. Maddison, “A development and evaluation process for mhealth interventions: Examples from New Zealand," J. Health Commun., vol. 17, no. SUPPL. 1, pp. 11-21, 2012.

[24] S. Michie, L. Yardley, R. West, K. Patrick, and F. Greaves, "Developing and Evaluating Digital Interventions to Promote Behavior Change in Health and Health Care: Recommendations Resulting From an International Workshop," J. Med. Internet Res., vol. 19, no. 6, Jun. 2017,

[25] V. Venkatesh, F. D. Davis, V. Venkatesh, and F. D. Davis, "A Theoretical Extension of the Technology Acceptance Model : Four Longitudinal Field Studies," Manage. Sci., vol. 46, no. 2, pp. 186-204, 2000.

[26] K. L. Carman et al., "Patient and Family Engagement: A Framework For Understanding The Elements And Developing Interventions And Policies," Health Aff., vol. 32, no. 2, pp. 223-231, 2013,

[27] R. McCorkle et al., "Self-management: Enabling and empowering patients living with cancer as a chronic illness," CA. Cancer J. Clin., vol. 61, no. 1, pp. 50-62, 2011, [Online].

[28] T. PDQ Supportive and Palliative Care Editorial Board, "Cancer Pain (PDQ $\left.{ }^{\circledR}\right)$-Patient Version," National Cancer Institute, 2019.

[29] T. The cancer.net Editorial Board, "Managing CancerRelated Pain," Conquer Cancer: The ASCO Foundation, 2019. .

[30] T. Cancer Care Ontario, "How to Manage Your Pain," Cancer Care Ontario Patient Guide, 2016.

[31] medical and editorial content The American Cancer Society, "Facts About Cancer Pain," American Cancer Society Treatment \& Support, 2019. .

[32] "Cancer and Pain Control," Cancer Research UK, 2018. https://www.cancerresearchuk.org/aboutcancer/coping/physically/cancer-and-pain-control/aboutcancer-pain.

[33] F. Maghnati and K. C. Ling, "Exploring the Relationship between Experiential Value and Usage Attitude towards Mobile Apps among the Smartphone Users," Int. J. Bus. Manag., vol. 8, no. 4, pp. 1-9, 2013.

[34] Agency for Healthcare Research and Quality, "Assess, Select, and Create Easy-to-Understand Materials," 2015. [Online]. Available: https://www.ahrq.gov/sites/default/files/wysiwyg/professiona 1s/quality-patient-safety/quality-resources/tools/literacytoolkit/healthlittoolkit2_tool11.pdf.

[35] K. T. Win, N. M. Hassan, H. Oinas-Kukkonen, and Y. Probst, "Online Patient Education for Chronic Disease Management: Consumer Perspectives," J. Med. Syst., vol. 40, no. 4, pp. 1-13, 2016, doi: 10.1007/s10916-016-0438-0.

[36] S. J. Shoemaker, M. S. Wolf, and C. Brach, "Development of the Patient Education Materials Assessment Tool (PEMAT): A new measure of understandability and actionability for print and audiovisual patient information," Patient Educ. Couns., vol. 96, no. 3, pp. 395-403, 2014.

[37] Q. A. for H. R. And, "PEMAT for Printable Materials (PEMAT-P) Agency for Healthcare Research and Quality," 2013. https://www.ahrq.gov/professionals/preventionchronic-care/improve/self-mgmt/pemat/pemat-p.html (accessed Jun. 08, 2019).

[38] Health Literacy Innovations, "Health Litearcy Innovations. Newsletter, Volume 1, Issue 1. Available at:," 2019. http://www.healthliteracyinnovations.com/newsletter/ (accessed Jun. 24, 2019).

[39] S. Badarudeen and S. Sabharwal, "Assessing readability of patient education materials: Current role in orthopaedics," Clin. Orthop. Relat. Res., vol. 468, no. 10, 2010.

[40] Y. L. Joffe H, "4. Content and Thematic Analysis," in Marks DF, Yardley L, Ed. Research methods for clinical \& health psychology., London: SAGE Publications Ltd, 2004, p. 56.

[41] R. J. Gatchel, Y. B. Peng, M. L. Peters, P. N. Fuchs, and D. C. Turk, "The Biopsychosocial Approach to Chronic Pain: Scientific Advances and Future Directions," Psychol. Bull., vol. 133, no. 4, pp. 581-624, 2007.

[42] S. S. Gorin et al., "Meta-Analysis of Psychosocial Interventions to Reduce Pain in Patients With Cancer," $J$. Clin. Oncol., vol. 30, no. 5, pp. 539-547, 2012.

[43] P. Menezes et al., "Use of a Mobile Phone App to Treat Depression Comorbid With Hypertension or Diabetes: A Pilot Study in Brazil and Peru," JMIR Ment. Heal., vol. 6, no. 4, p. e11698, 2019, doi: 10.2196/11698.

[44] S. Michie, C. Abraham, C. Whittington, J. McAteer, and S. Gupta, "Effective Techniques in Healthy Eating and Physical Activity Interventions: A Meta-Regression," Heal. Psychol., vol. 28, no. 6, pp. 690-701, 2009.

[45] R. R. Edwards et al., "Patient phenotyping in clinical trials of chronic pain treatments," Pain, vol. 157, no. 9, pp. 1851-1871, 2016.

[46] J. F. M. Van Dijk, S. C. J. M. Vervoort, A. J. M. Van Wijck, C. J. Kalkman, and M. J. Schuurmans, "Postoperative patients' perspectives on rating pain: a qualitative study," Int. J. Nurs. Stud., vol. 53, pp. 260-269, 2016. 